\title{
P.P. Ахунов
}

\section{Анализ полосковых структур радиоэлектронных устройств с уменьшенными вычислительными затратами}

\footnotetext{
Приведены результаты вычисления значений погонных коэффициентов электростатической и электромагнитной индукции для связанной микрополосковой линии. Результаты получены с использованием алгоритмов решения систем линейных алгебраических уравнений итерационным методом с предобусловливанием. Для предобусловливания использован формат хранения разреженных матриц, что позволило уменьшить вычислительные затраты. Приведено сравнение полученных результатов с результатами других авторов. Сравнение показало согласованность результатов.

Ключевые слова: полосковые структуры, метод моментов, система линейных алгебраических уравнений, итерационный метод, предобусловливание, погонная матрица. doi: $10.21293 / 1818-0442-2017-20-2-96-99$
}

Использование полосковых структур позволяет разрабатывать более совершенные радиоэлектронные устройства (РЭУ) различного назначения, а анализ связей в полосковых структурах важен для разработки РЭУ, в том числе с учетом электромагнитной совместимости. Для разработки РЭУ используется предварительное компьютерное моделирование с целью снизить финансовые и временные затраты. В общем случае в основе моделирования лежит численный анализ, требующий построения математической модели исследуемого объекта с помощью решения уравнений Максвелла. Основные численные методы, применяемые в моделировании ЭМС: методы конечных разностей во временной области [1]; метод моментов (МоМ) [2]; метод конечных элементов [3]; метод конечного интегрирования [4]; метод матрицы линий передачи [5], а также так называемые «гибридные» методики [6].

Процесс построения математической модели состоит из нескольких этапов [7]: постановка задачи; аналитическая обработка; дискретизация (сегментация) модели; вычисление элементов СЛАУ и её решение СЛАУ; обработка результатов.

Описанные этапы не являются независимыми. Метод дискретизации определяет затраты на вычисление элементов СЛАУ, а также её порядок и свойства (обусловленность, плотность), что, в конечном итоге, определяет выбор метода её решения (прямой или итерационный). От результатов, полученных на предыдущих этапах, зависят и способы вычисления требуемых параметров и характеристик моделируемого объекта/системы.

На втором этапе широко используются электродинамический и квазистатический (ТЕМ-аппроксимация) подходы к решению уравнений Максвелла. На третьем этапе при решении задач ЭМС широко используется метод моментов, использующий «поверхностный» подход, в соответствии с которым в качестве неизвестного выступает распределение плотности поверхностного заряда на проводящих поверхностях исследуемого объекта/системы [8]. Найденный поверхностный заряд рассматривается как источник, возбуждающий поле во всей расчетной области. Таким образом, при использовании МоМ неизвестная функция определена на поверхности, а не в объеме (как при использовании, например, методов конечных разностей, элементов и объемов), что уменьшает требования к вычислительным ресурсам. Получаемая при этом матрица СЛАУ является плотной и плохо обусловленной, что требует построения эффективных предобусловливателей для ускорения решения при использовании итерационных методов. Поскольку один из самых трудоёмких этапов приходится на решение СЛАУ [8], то актуально совершенствование методов их решения.

Автором были предложены алгоритмы, использующие формат хранения разреженных матриц [9, 10]. За счет этого удалось снизить вычислительные затраты на решение СЛАУ. Причем были снижены как время решения, так и требуемая память компьютера. Однако ранее не был выполнен анализ влияния полученного уменьшения вычислительных затрат на точность получаемых результатов.

Цель работы - сравнение результатов вычисления значений погонных коэффициентов электростатической и электромагнитной индукции для связанной микрополосковой линии с помощью алгоритмов, предложенных в $[9,10]$, с результатами других авторов.

Итерационные методы решения СЛАУ

Существует два класса методов решения СЛАУ: точные и итерационные методы. Для точных методов основные вычислительные затраты пропорциональны $N^{3}$ ( $N$ - порядок матрицы), что ограничивает использование таких методов при больших порядках матрицы СЛАУ. При этом для итерационных методов вычислительные затраты пропорциональны $N_{\mathrm{it}} \cdot N^{2}$ ( $N_{\mathrm{it}}-$ количество итераций). Из этого следует, что при $N_{\text {it }}<N$ (а это часто имеет место) использовать итерационные методы выгоднее. Однако итерационные методы не лишены недостатков. Основная их проблема - медленная сходимость. Скорость сходимости зависит от спектральных свойств матрицы СЛАУ, от обусловленности и даже от начального 
приближения. Для ускорения сходимости в итерационных методах используют предобусловливание [11]. Принцип предобусловливания заключается в преобразовании системы $\mathbf{A x}=\mathbf{b}$ к алгебраически эквивалентной системе $\tilde{\mathbf{A}} \tilde{\mathbf{x}}=\tilde{\mathbf{b}}$, которая имеет меньшую обусловленность. Условно методы предобусловливания делят на два вида: явные и неявные. При явном предобусловливании исходная система преобразуется к системе $\mathbf{M}^{-1} \mathbf{A x}=\mathbf{M}^{-1} \mathbf{b}$. При неявном $-\mathbf{K} \mathbf{M A x}=\mathbf{M b}$. Неявное предобусловливание не требует вычисления $\mathbf{M}^{-1}$, но требует решения СЛАУ в каждой итерации. Однако, несмотря на это, использование неявного предобусловливания все равно выгоднее [11]. Большая часть неявных методов основана на представлении $\mathbf{M}=\mathbf{L} \mathbf{U}$, где $\mathbf{L}$ и $\mathbf{U}$ получены путем LU-разложения из матрицы $\mathbf{A}_{s}$, обычно получаемой из исходной матрицы А путем уменьшения количества ненулевых элементов (предфильтрации). Как правило, матрица $\mathbf{A}_{s}$ является разреженной, и для её хранения можно использовать формат хранения разреженных матриц [12]. Один из самых эффективных форматов - разреженный строчный формат (Compressed Storage Row, CSR). Ненулевые элементы матрицы в формате CSR хранятся с помощью векторов: Values - значения ненулевых элементов; Columns - индексы столбцов; RowIndex - вектор указателей на ненулевые элементы, с которых начинается очередная строка. Однако метод LU-разложения предполагает неконтролируемое добавление новых элементов. Между тем существуют методы, которые контролируют добавление новых элементов. Один из таких методов, который не требует добавления ненулевых элементов, - это $\operatorname{ILU}(0)$-разложение. Для ясности изложения далее приведены 2 алгоритма ILU(0)-разложения, предложенные в работах $[9,10]$.

Алгоритм 1 - ILU(0)-разложение с использованием формата CSR и дополнительного вектора Diag (вектор указателей на диагональные элементы):

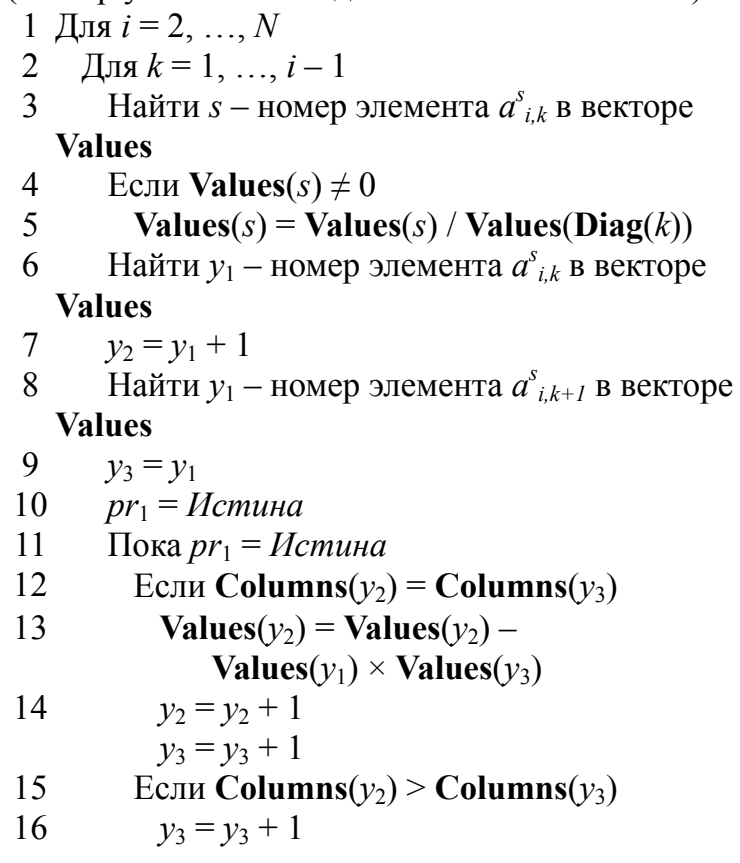

21 Увеличить $k$

\section{2 Увеличить $i$}

В строках 3, 6, 8 производится поиск ненулевых элементов матрицы $\mathbf{A}_{s}$. Переменная $p r_{1}$ сигнализирует, когда достигнут конец строки $i$ или $k$ в матрице и требуется прервать цикл. Переменные $y_{2}$ и $y_{3}$ определяют текущей элемент в первой (i-й) и второй $(k$-й) строках соответственно. Переход по элементам строк в матрице осуществляется в цикле по следующему принципу: сравниваются индексы элементов двух текущих строк, индекс с меньшим значением инкрементируется (строки 15-18), если индексы элементов равны (т.е. существуют ненулевые элементы в двух строках текущего столбца), то выполняется операция и значения индексов обеих переменных инкрементируются (строки 12-14).

Алгоритм 2 - ILU(0)-разложение с использованием формата CSR с двумя дополнительными векторами:

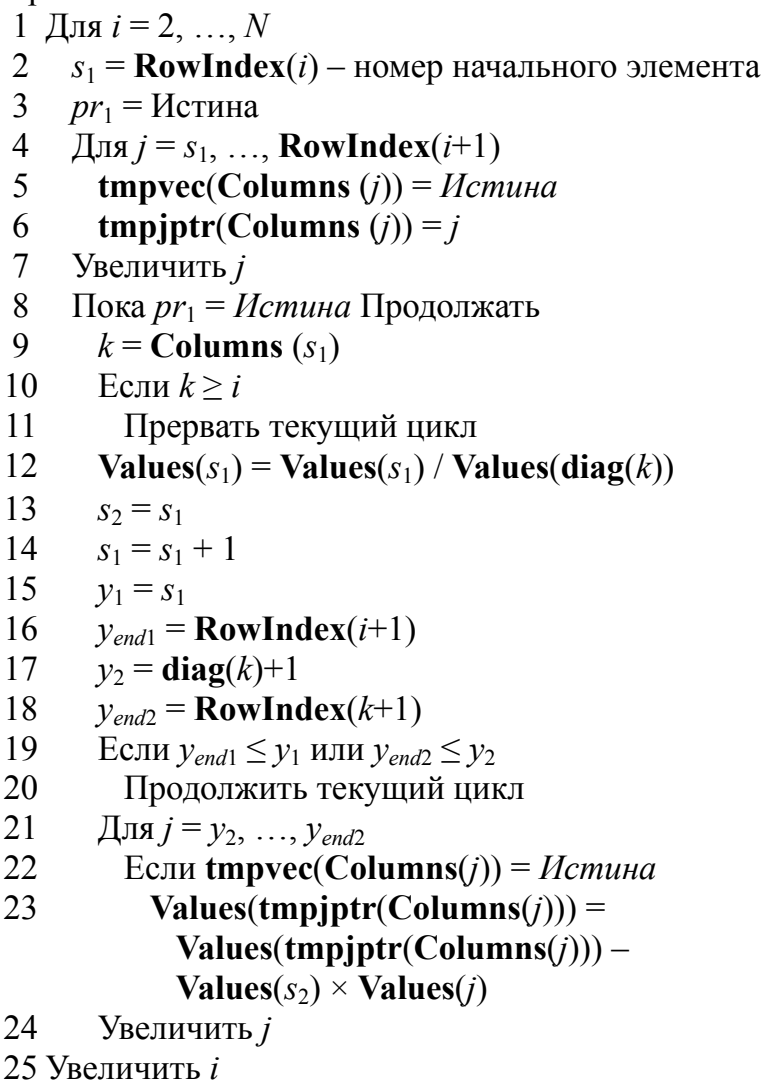

В векторе tmpvec хранится значение Истина, если элемент матрицы $\mathbf{A}_{s}$ ненулевой, а в векторе tmpjptr хранится адрес этого ненулевого элемента. Переменные $y_{\text {end } 1}$ и $y_{\text {end } 2}$ определяют начало и конец строки.

\section{Вычислительный эксперимент}

Предложенные алгоритмы универсальны, а метод моментов с предложенными алгоритмами позво- 
ляет анализировать структуры с произвольным числом проводников и диэлектриков с границами произвольной формы. Однако опубликованы результаты (с которыми можно сравнить свои) только для относительно простых структур. Поэтому в качестве анализируемой структуры взята широко используемая связанная микрополосковая линия, поперечное сечение которой приведено на рис. 1. Использовано 2 набора параметров структуры, которые взяты из работ $[13,14]$. Для наглядности параметры сведены в табл. 1. Для сравнения использовались значения погонных коэффициентов электростатической $\left(C_{11}\right.$, $\left.C_{12}\right)$ и электромагнитной $\left(L_{11}, L_{12}\right)$ индукций.

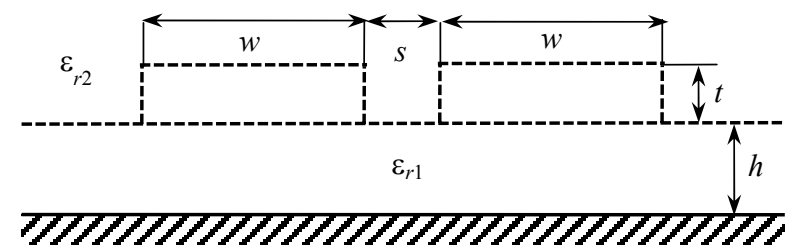

Рис. 1. Поперечное сечение связанной микрополосковой линии

Таблица 1

Значения параметров для

связанной микрополосковой линии

\begin{tabular}{|c|c|c|}
\hline \multirow{2}{*}{ Параметр } & \multicolumn{2}{|c|}{ Набор } \\
\cline { 2 - 3 } & 1 & 2 \\
\hline$w$, мм & 0,9 & 3 \\
\hline$t$, мм & 0,05 & 1 \\
\hline$s$, мм & 0,8 & 2 \\
\hline$h$, мм & 1 & 1 \\
\hline$\varepsilon_{r 1}$ & 10 & 2 \\
\hline$\varepsilon_{r 2}$ & 1 & 1 \\
\hline
\end{tabular}

За счет использования алгоритмов 1 и 2 получено ускорение решения СЛАУ. Так, алгоритм 1 быстрее до 1,89 раза по сравнению с алгоритмом без использования формата CSR [9] (для набора 2 при $N=1100$ время вычисления 1,57 с против 2,97 с), а алгоритм 2 быстрее до 1,59 раза относительно алгоритма 1 [10] (для набора 1 при $N=1950$ время вычисления 1,52 с против 2,41 с). При этом коэффициент сжатия матрицы $\mathbf{A}_{s}$ достигал 2,66 раза. (Использовался компьютер с параметрами: платформа AMD FX(tm)-8320 Eight-Core Processor; частота процессора - 3,50 ГГц; объем ОЗУ - 16 Гбайт; число ядер - 8; операционная система - Windows 7 х64).

Учащением сегментации структуры были получены СЛАУ с матрицами разных порядков. В табл. 2 приведено сравнение значений погонных коэффициентов электростатической и электромагнитной индукций, полученных с использованием алгоритмов 1 и 2, с результатами других авторов.

Из табл. 2 видно, что для обоих наборов параметров результаты для разных алгоритмов не отличаются, а из-за учащения сегментации значения коэффициентов электростатической индукции отличаются несущественно $\left(C_{12}\right.$ менее $4 \%, C_{11}$ менее $1 \%$ ), тогда как электростатической - ещё меньше. Результаты алгоритмов в целом согласуются с ре- зультатами других авторов (отклонения для $C_{11}$ не более $9 \%$, для $C_{12}-$ не более $24 \%$, для $L_{11}-$ не более $7 \%$, для $L_{12}-$ не более $20 \%$ ).

Для набора 1 разница объяснима различными методами решения задачи: в работе [13] используется метод конформных отображений. Для набора 2 в работах [14-16] также используется метод моментов, поэтому согласованность результатов выше, а разница объяснима разной сегментацией. Наибольшее расхождение наблюдается с результатами из работы [16], в которой использованы трехмерное моделирование и RWG-базисные функции. Отличие результатов работ $[14,15]$ объяснимо различием используемых тестовых функций.

Т а бли ц а 2

Значения погонных коэффициентов связанной микрополосковой линии

\begin{tabular}{|c|c|c|c|c|c|}
\hline \multirow[t]{2}{*}{ Источник } & \multicolumn{5}{|c|}{ 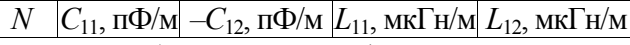 } \\
\hline & \multicolumn{5}{|c|}{ Набор параметров 1} \\
\hline \multirow{4}{*}{$\begin{array}{l}\text { Алго- } \\
\text { ритм } 1\end{array}$} & 650 & 146,65 & 23,71 & 0,421 & 0,099 \\
\hline & 975 & 146,80 & 23,98 & 0,421 & 0,099 \\
\hline & 1300 & 146,74 & 24,29 & 0,421 & 0,099 \\
\hline & 1950 & 147,03 & 24,33 & 0,421 & 0,099 \\
\hline \multirow{4}{*}{$\begin{array}{l}\text { Алго- } \\
\text { ритм } 2\end{array}$} & 650 & 146,65 & 23,71 & 0,421 & 0,099 \\
\hline & 975 & 146,80 & 23,98 & 0,421 & 0,099 \\
\hline & 1300 & 146,74 & 24,29 & 0,421 & 0,099 \\
\hline & 1950 & 147,03 & 24,33 & 0,421 & 0,099 \\
\hline \multicolumn{2}{|l|}{ [13] } & 159,81 & 20,81 & 0,422 & 0,100 \\
\hline \multicolumn{6}{|c|}{ Набор параметров 2} \\
\hline \multirow{4}{*}{$\begin{array}{l}\text { Алго- } \\
\text { ритм } 1\end{array}$} & 68 & 90,73 & 8,86 & 0,200 & 0,030 \\
\hline & 220 & 90,64 & 9,11 & 0,198 & 0,030 \\
\hline & 330 & 90,90 & 9,14 & 0,198 & 0,030 \\
\hline & 1100 & 91,35 & 9,18 & 0,198 & 0,030 \\
\hline \multirow{4}{*}{$\begin{array}{l}\text { Алго- } \\
\text { ритм } 2\end{array}$} & 68 & 90,73 & 8,86 & 0,200 & 0,030 \\
\hline & 220 & 90,64 & 9,11 & 0,198 & 0,030 \\
\hline & 330 & 90,90 & 9,14 & 0,198 & 0,030 \\
\hline & 1100 & 91,35 & 9,18 & 0,198 & 0,030 \\
\hline [14] & & 92,36 & 8,49 & 0,198 & 0,030 \\
\hline [15] & & 92,24 & 8,50 & 0,198 & 0,030 \\
\hline [16] & & 91,89 & 7,02 & 0,186 & 0,024 \\
\hline
\end{tabular}

\section{Заключение}

В работе выполнено сравнение значений погонных коэффициентов электростатической и электромагнитной индукции, полученных с помощью алгоритмов, позволяющих усовершенствовать решение СЛАУ, с результатами других авторов, на примере связанной микрополосковой линии. Показано, что использование данных алгоритмов позволяет уменьшить вычислительные затраты при контролируемой точности значений вычисляемых параметров. Таким образом, данные алгоритмы могут быть эффективно использованы при анализе других полосковых структур.

Исследование выполнено за счет проекта 8.9562.2017/8.9 Минобрнауки Российской Федерации.

\section{Благодарность}

Автор благодарен рецензенту за ценные замечания. 


\section{Литература}

1. Сосунов Б.В. Применение метода конечных разностей временной области в задачах дифракции радиоволн / Б.В. Сосунов, А.А. Тимчук // Вопросы ЭМС и расчета антенн и радиолиний: ВАС. - СПб., 1994. - С. 220-226.

2. Харрингтон Р.Ф. Применение матричных методов к задачам теории поля // ТИИЭР. - 1967. - № 2. - С. 5-19.

3. Silvester P. Finite element solution of saturate magnetic field problems / P. Silvester, M. Chari // IEEE Trans. Power Appar. Syst. - 1970. - Vol. 89. - № 7. - P. 1642-1651.

4. Weiland T.A. Discretization Method for the Solution of Maxwell's Equations for Six-Component Fields / T.A. Weiland // Electronics and Communications AEUE.1977. - Vol. 31(3). - P. 116-120.

5. Johns P.B. Numerical solution of 2-dimensional scattering problems using a transmission line matrix / P.B. Johns, R.L. Beurle // Proceedings of the IEEE. - 1971. - Vol. 118(9). P. 1203-1208.

6. Агапов С.В. Электронные САПР для моделирования электромагнитных излучений от межсоединений печатных плат / С.В. Агапов // Проблемы электромагнитной совместимости технических средств: сб. докл. Всерос. симпозиума. - М., 2002. - С. 11-13.

7. Григорьев А.Д. Методы вычислительной электродинамики. - М.: Физматлит, 2013. - 430 с.

8. Газизов Т.Р. Уменьшение искажений электрических сигналов в межсоединениях и влияний преднамеренных силовых электромагнитных воздействий: дис. ... д-ра техн. наук. - Томск, 2010. - $351 \mathrm{c}$.

9. Ахунов Р.Р. Форматы хранения разреженных матриц и ускорение решения СЛАУ с плотной матрицей итерационными методами / Р.Р. Ахунов, С.П. Куксенко, В.К. Салов, Т.Р. Газизов // Численные методы и вопросы организации вычислений. XXV Зап. научн. семин. ПОМИ. 2012. - T. 405(25). - C. 24-39.

10. Ахунов Р.Р. Усовершенствование алгоритма $\operatorname{ILU}(0)-$ разложения, использующего разреженный строчный формат / Р.Р. Ахунов, С.П. Куксенко, В.К. Салов, Т.Р. Газизов // Численные методы и вопросы организации вычислений. XXV Зап. научн. семин. ПОМИ. - 2012.T. 405(25). - C. 40-53.

11. Газизов Т.Р. Оптимизация допуска обнуления при решении СЛАУ итерационными методами с предобусловливанием в задачах вычислительной электродинамики / Т.Р. Газизов, С.П. Куксенко // Электромагнитные волны и электронные системы. - 2004. - № 8. - С. 26-28.

12. Писсанецки С. Технология разреженных матриц: Пер. с англ. - М.: Мир, 1988. - 410 с.
13. Sychev A.N. Modeling of the coupled microstrip lines with using numerical conformal transformations / A.N. Sychev, M.A. Chekalin, V.A. Shestakov, S.M. Struchkov // European Microwave Week 2013 Conf. Proc.-Eur. MC 2013. - 6-11 Oct. 2013, Nuremberg, Germany. - P. 11071110.

14. Venkataraman J. Analysis of Arbitrarily Oriented Microstrip Transmission Lines in Arbitrarily Shaped Dielectric Media Over a Finite Ground Plane / J. Venkataraman, S.M. Rao, A.R. Djordjevic et al. // IEEE Tran. Microwave Theory Tech. - 1985. - Vol. 33 (10). - P. 952-960.

15. Weeks W.T. Calculation of Coefficients of Capacitance of Multiconductor Transmission Lines in the Presence of a Dielectric Interface // IEEE Tran. Microwave Theory Tech. 1970. - Vol. 18 (1). - P. 35-43.

16. Sarkar T.K. The Electrostatic Field of Conducting Bodies in Multiple Dielectric Media / T.K. Sarkar, R.F. Harrington // IEEE Tran. Microwave Theory Tech. - 1984.Vol. 32 (11). - P. 1441-1448.

\section{Ахунов Роман Раисович}

Инженер-исследователь науч.-иссл. лаб. «Безопасность и электромагнитная совместимость радиоэлектронных средств» ТУСУРа

Тел.: +7-913-888-12-13

Эл. почта: arr_garant@mail.ru

Akhunov R.R.

Analysis of strip structures of radioelectronic devices with reduced computational costs

The results of calculating the values of the per unit-of-length coefficients of electrostatic and electromagnetic induction for a coupled microstrip line are presented. The results are obtained using algorithms for linear systems solving by an iterative method with preconditioning. For preconditioning, the format for storing the sparse matrices was used that was permitting to reduce computational costs. The results obtained are compared with the results of other authors. The comparison showed consistency of the results.

Keywords: strip structures, method of moments, linear algebraic system, iterative method, preconditioning, per unit-oflength matrix. 